\title{
Separation of Gas and Vapour using Ethylene-Octane Polymer Membranes
}

\section{Kinoma Naranis*}

Ph.D Student, Institute for Anthropologica, Croatia

*Corresponding author: Kinoma Naranis, Ph.D Student, Institute for Anthropologica, Ljudevita Gaja 32, 10000 Zagreb, Croatia, Tel: 385-1-5535133; E-mail: kinoma_naran@inantro.hr

Rec date: June 15, 2014; Acc date: July 14, 2014; Pub date: July 22, 2014

Copyright: (c) 2014 Naranis K. This is an open-access article distributed under the terms of the Creative Commons Attribution License, which permits unrestricted use, distribution, and reproduction in any medium, provided the original author and source are credited.

\begin{abstract}
Organic vapors are additional pervious than permanent gases within the composite membranes, with solvent and resolving permeability being concerning orders of magnitude beyond permanent gas permeableness. Gas and vapor transport properties were studied in mixed matrix membranes containing elastomeric ethylene-octene polymer (EOC or poly(ethylene-co-octene)) with 3 styles of carbon fillers: virgin or modify multi-walled carbon nanotubes (CNTs) for the open-chain organic compound (hexane), hydrocarbon (toluene), alcohol (ethanol), likewise as water for the representative samples. Gas permeableness of EOC lies between that of the additional pervious PDMS and also the less pervious semi-crystalline polythene and plastic. The results of the carbon-filled membranes supply views for application in gas/vapor separation with improved mechanical resistance.
\end{abstract}

Keywords: Gas; Vapour; Ethylene-Octane polymer membrane; Stored fuels

\section{Introduction}

Storage and handling of fuel, and conjointly supplying of cars, involve associate degree outflow of fuel vapors into the atmosphere [1]. Supplying stations will solve this downside by debilitating the vapors into the tanks. Hence, since the Eighties there was an endeavor to capture these hydrocarbons and to recycle them. Presently, the method of volatile organic compounds (VOCs) removal from the air is allotted by totally different strategies. The foremost wide used technique is that the absorption of VOCs in $\mathrm{C}$ or in a very appropriate solvent. Even so, the absorption may be a discontinuous method wherever periodic replacement of the absorbent is required and, therefore, it's connected with the danger of the increase of waste material dumps and waste production [2].

Conventionally, toughness has been characterized by the Izod impact test. The addition of cellulose microfibers in PLA/EVA-GMA system modifies the rheological behavior, since complex viscosity increased in presence of fibers and decreased with an increase in frequency. Disintegration tests showed that the addition of EVA-GMA influence the PLA disintegration process and after 21 days in composting conditions, blends and composites showed faster degradation rate in comparison with neat PLA due to the different morphologies induced by the presence of EVA-GMA and CF phases able to allow faster water diffusion and an efficient PLA degradation process.

In distinction, membrane separation constitutes a safer and additional advanced technique, and membrane separations for VOCs removal are characterized by a high potency. The most blessings offered by membrane processes are [3-5] energy savings, environmental friendliness, easy handling, continuous process, compact style and tiny footprint (Figure 1).

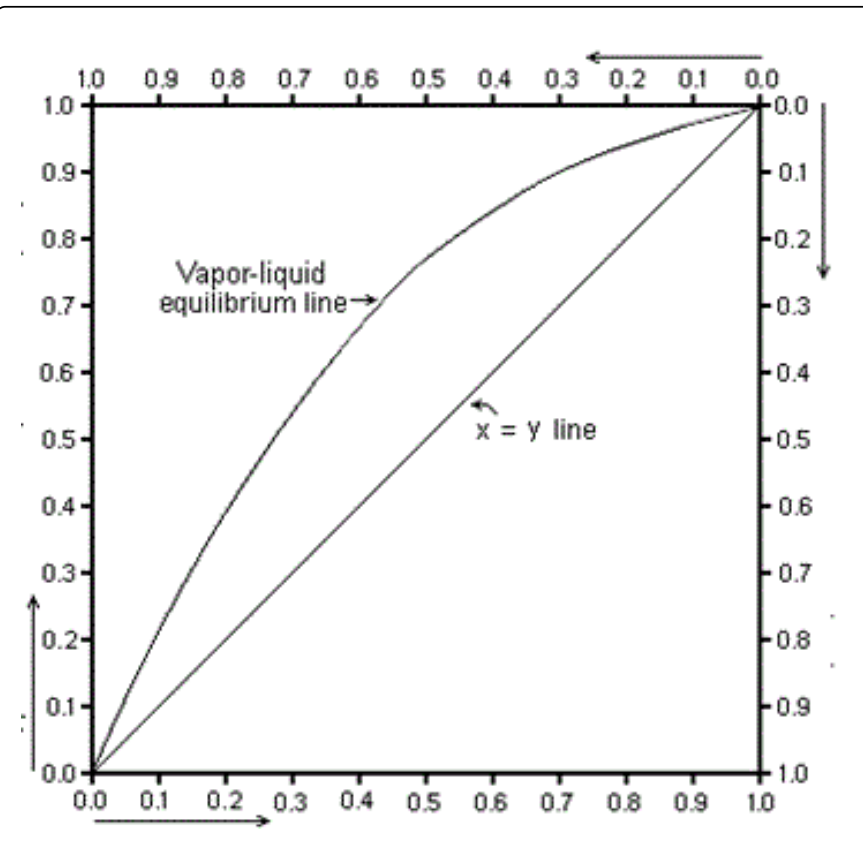

Figure 1: Separation phases in volatile equilibrium.

Among compound membranes used for VOCs separations [6], those supported polydimethylsiloxane (PDMS) predominate [7-9]. The chemical stability of PDMS isn't decent and it conjointly swells powerfully [10] once it's connected with organic vapors. Therefore, there's still a probe for various materials to PDMS with comparably appropriate characteristics and higher stability. within the past few decades, varied membrane materials have been tested, such as, for instance, poly(ether-amide) block-copolymer (PEBA), polyvinylidene halide (PVDF), high-free volume amorphous glassy perfluoropolymers [11], cross-linked fluorinated or poly(amide-imide) polymers [12,13], and semi-crystalline polyolefins [14]. 


\section{Advantages of Octane Polymer Membrane [15]}

- Because membrane processes can separate at the molecular scale up to a scale at which particles can actually be seen, this implies that a very large number of separation needs might actually be met by membrane processes.

- Membrane processes generally do not require a phase change to make a separation (with the exception of evaporation). As a result, energy requirements will be low unless a great deal of energy needs to be expended to increase the pressure of a feed stream in order to drive the permeating component(s) across the membrane.

- Membrane processes present basically a very simple flowsheet. There are no moving parts (except for pumps or compressors), no complex control schemes, and little ancillary equipment compared to many other processes. As such, they can offer a simple, easy-tooperate, low maintenance process option.

- Membranes can be produced with extremely high selectivities for the components to be separated. In general, the values of these selectivities are much higher than typical values for relative volatility for distillation operations.

- Because of the fact that a very large number of polymers and inorganic media can be used as membranes, there can be a great deal of control over separation selectivities.

- Membrane processes are able to recover minor but valuable components from a main stream without substantial energy costs.

- Membrane processes are potentially better for the environment since the membrane approach require the use of relatively simple and non-harmful materials.

\section{Evaluation of Polymer to Separate the Phases}

The appropriate choice of compound will guarantee decent chemical resistance of the ultimate membrane for permeation of gases or vapors. During this context, polyolefin are often thought-about as potential candidates for membrane applications. EOC was chosen within the gift work because it is additional pervious than the semicrystalline analogous polyolefin polythene and plastic according antecedently $[16,17]$.

In flat sheet configuration, membranes are typically subjected to compression forces. These forces could become vital in aggressive applications, like in membranes for gas separation or for reverse diffusion. Within the case of heterogenous porous supports, such compression forces are translated into a tensile force within the dense skin. Therefore, data of the material's tensile properties is very important. The latter is especially relevant within the case of hollow fiber membranes, within which the interior pressure is translated like a shot into a tensile force on the membrane wall $[18,19]$.

In order to beat the limitation of both polymeric and inorganic membranes, Mixed Matrix Membranes (MMMs), consisting of a dispersion of filler particles among a compound matrix, are wide investigated to beat the upper-bound trade-off limit of the compound membranes likewise because the main drawbacks, like brittleness and lack of reproducibility related to inorganic membranes [20]. Thus, these systems are doubtless appropriate to mix the exclusive blessings in separation performance of each inorganic and compound materials. Chemical structure, surface chemistry, size, and ratio are the foremost necessary variables for filler choice, whereas filler-polymer compatibility and filler distribution are the key points for a good MMM preparation [21,22]. Theoretical models are wont to predict and interpret the gas transport properties in MMMs. A basic approach uses the permeableness of the 2 phases and also the filler concentration (Maxwell's model). Some modifications were projected so as to require into consideration the filler ratio, likewise because the contribution of the interface polymer/filler [18]. Porous fillers are wont to enhance transport rates, though it's been incontestable that dense fillers may have this result if the polymer-particle interface plays a very important role [19].

It was shown that the catalyst structure and reaction conditions, like the reaction temperature and concentration of activator (MAO), have substantial influence on the polymerization activities and molecular weights and microstructures of the resulting polymers (Figure 2).

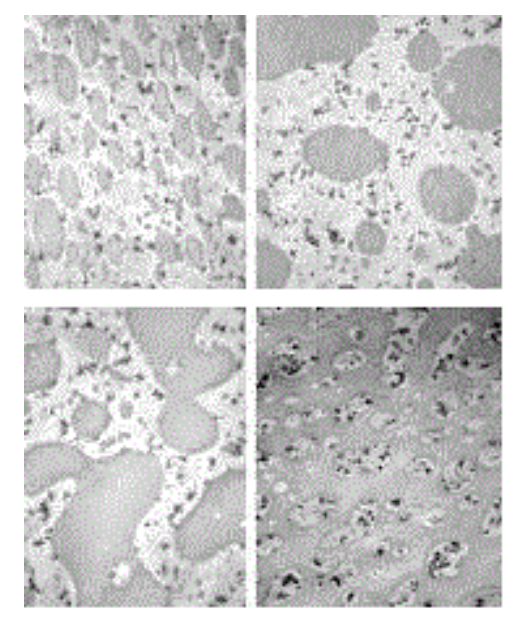

Figure 2: Octane polymer layers in different dimension.

Carbon fillers, like carbon nanotubes (CNTs) and carbon fibers (CFs), are terribly attention-grabbing materials for Nano composites preparation with a high reinforcing potential, already exploited in several applications (e.g., region and transportation). CNTs or CFs ar typically additional to compound matrixes for mechanical reinforcement, and conjointly for a rise of their electrical and thermal conduction [20] or else, changes of the electrical conduction of the material elicited by exposure to gases and vapors [23], by everchanging the close temperature [2], or by mechanical deformation of the membrane $[1,7]$, build these materials doubtless appropriate for sensing element applications. Within the case of membrane separation processes involving flammable gases, increased electrical conduction of membranes improves the method safety, preventing electrical charge accumulation. Finally, the electrical conduction of the membranes is additionally associate degree indirect live of the dispersion of the filler within the matrix.

The advantage of all higher than mentioned properties rely well on the state of filler dispersion within the compound matrix. A mixing of fillers into compound matrices in compound soften is commonly used for composite fabrication. Carbon-based materials, like CNTs or CFs, are typically incompatible with polymers, resulting in filler agglomeration in compound matrices instead of individualization of the filler particles to boost the dispersion state of filler, high-energy strategies, such ultrasound treatment of the filler spread within the compound resolution, are typically used. Higher results are achieved by the precipitation of the compound from resolution employing a non-solvent [24] to stop filler alleviation and aggregation than by the 
solvent casting technique [5]. This could conjointly apply within the case of ethylene-octene polymer (EOC), that dissolves in cold solvent however isn't soluble in common solvents, like propanone.

In some cases, CNTs and CFs are reported to boost the transport properties in dense rubbery membranes or in glassy polymer membranes [13]. They have conjointly been used in their neat type as materials for water purification and gas separation membranes. within the gift manuscript, the dispersion of those carbon fillers in EOC are investigated, with explicit interest for mechanical properties and the gas and vapor transport properties of the ensuing MMMs.

\section{Membrane Preparation}

Composites of MWCNT associate degreed CF fillers in an EOC matrix were ready by dispersion of the fillers within the compound resolution, exploitation the ultra-sonication technique. Firstly, an answer of $5 \mathrm{wt}$. the concerns EOC in solvent was ready and calculated amounts of fillers were additional to yield composites containing a pair of $5,10,15,20,25,30$, and $35 \mathrm{wt}$ capitalize on fillers within the final mix [18].

\section{Mechanical Tests}

Mechanical properties of all membranes were allotted on a Zwick/ Roell Universal Testing machine (single column, model Zwick Z2.5, Ulm, Germany) equipped with a fifty $\mathrm{N}$ most load cell and with gas clamps. The clamps surface was coated with associate degree adhesive rubber to avoid slippy of the membrane strips. The membrane samples were dig strips of $5 \mathrm{~mm}$ dimension. The effective membrane strips length was $30 \mathrm{~mm}$ (i.e., the gap between the clamps) [9]. The strips thickness was measured with the digital micrometer in a minimum of 5 points and also the average worth was used.

\section{Gas and Vapor Permeableness Measurements}

The permeation experiments were performed on a hard and fast volume/pressure increase instrument, made by GKSS (Geesthacht, Germany). The feed force per unit area was set at one bar (the actual worth was browse with a resolution of zero. 1 mbar); the permeate pressure was measured in the vary from zero to most, $13.3 \mathrm{mbar}$, with a resolution of 0.001 mbar. An equivalent protocol was followed for the neat compound membrane likewise as for all hybrid samples [11].

The gases were forever tested within the same order $\left(\mathrm{He}, \mathrm{H}_{2}, \mathrm{~N}_{2}\right.$, $\mathrm{O}_{2}, \mathrm{CH}_{4}$, and $\mathrm{CO}_{2}$ ), though it had been verified by continuation a activity cycle that if sufficiently long vacuum was applied to utterly take away the previous gas, that the activity order for these materials was tangential [24]. Feed pressure, permeate pressure, and temperature are ceaselessly recorded throughout every activity run. The temperature was controlled at a relentless temperature of $25 \pm$ $1^{\circ} \mathrm{C}$.

\section{Membrane Morphology}

The samples with modify CNTs had a rough surface, indicating poor dispersion of the fillers as giant aggregates. The untreated CNTs yielded sleek and consistent samples, however the presence of fibrous structures within the SEM pictures, at comparatively low magnification, indicates that the CNTs are gift within the type of bundles. The conduction sharply will increase higher than close to ten wt. capitalize on CNT or CF within the EOC matrix. Though absolutely the values are not up to those of EOC composites with CNTs functionalized with hyper branched polythene, the high conduction is associate degree indirect confirmation of the great dispersion of the carbon fillers within the compound matrix.

\section{Conclusions}

Gas associate degreed vapor transport measurements on ethyleneoctene polymer membranes show that the EOC is an organophilic material that may be doubtless used for membrane vapor separation from air or for a few gas separations involving mixtures of extremely condensable and lighter species.

Addition of carbon nanotubes and carbon fibers has comparatively very little result on the transport properties of sunshine gases and a modest result on the transport of vapors. For light-weight gases, the carbon fillers act as tight obstacles and also the gas permeableness slightly decreases in a very similar fashion as foreseen by the Maxwell model. For vapors, the behavior is opposite and also the addition of a tiny low quantity of carbon filler causes a rise in permeableness. At the same time, the CNTs and particularly the CFs enhance.

\section{References}

1. Éigenson AS (1973) Regularity in boiling point distribution of crude oil fractions. Chem. Technol. Fuels Oils 9: 3-8.

2. Kimmerle K, Bell CM (1988) Solvent recovery from air. J Membr Sci 36: 477-488.

3. Ravanchia MT, Kaghazchia T, Margarin A (2009) Application of membrane separation processes in petrochemical industry: A review. Desalination 235: 199-244.

4. Bernardo P, Drioli E, Golemme G (2009) Membrane gas separation: A review/state of the art. Ind Eng Chem Res 48: 4638-4663.

5. Leemann M, Eigenberger G, Strathmann H (1996) Vapour permeation for the recovery of organic solvents from waste air streams: Separation capacities and process optimization. J Membr Sci 113: 313-322.

6. Rebollar-Perez G, Carretier E, Lesage N, Moulin P (2011) Volatile organic compound (VOC) removal by vapor permeation at low VOC concentrations: Laboratory scale results and modeling for scale up. Membranes 1: 80-90.

7. Sysela P, Sindelara V, Kubonovab M, Friessb K, Hynekb V, et al., (2009) Membranes based on modified polyimides for gas and organic vapour separations. Desalination 236: 46-50.

8. Kim HJ, Nah SS, Min BR (2002) A new technique for preparation of PDMS pervaporation membrane for VOC removal. Adv Environ Res 6: 255-264.

9. Majumdar S, Bhaumik D, Sirkar KK (2003) Performance of commercialsize plasmapolymerized PDMS-coated hollow fiber modules in removing VOCs from N2/air. J Membr Sci 214: 323-330.

10. Sohn WI, Ryu DH, Oh SJ, Koo JK (2000) A study on the development of composite membranes for the separation of organic vapors. J Membr Sci 175: 163-170.

11. Liu Y, Feng X, Lawless D (2006) Separation of gasoline vapor from nitrogen by hollow fiber composite membranes for VOC emission control. J Membr Sci 271: 114-124.

12. Yampolskii Y, Pinnau I, Freeman B (2006) Materials Science of Membranes for Gas and Vapor separation. John Wiley \& Sons, New York, NY, USA.

13. Šindelář V, Sysel P, Hynek V, Friess K, Šípek M, et al., (2001) Transport of gases and organic vapors through membrane made of poly(amideimide)s crosslinked with poly(ethyleneadipate). Collect Czech Chem Commun 66: 533-540. 
Citation: Naranis K (2014) Separation of Gas and Vapour using Ethylene-Octane Polymer Membranes. J Bioproces Biotechniq 4: 169. doi: 10.4172/2155-9821.1000169

Page 4 of 4

14. Guizard C, Boutevin B, Guida F, Ratsimihety A, Amblard P, et al., (2001) VOC vapor transport properties of new membranes based on crosslinked fluorinated elastomers. Sep Purif Technol 22-23: 23-30.

15. Togawa J, Kanno T, Horiuchi JI, Kobayashi M (2001) Gas permeability modification of polyolefin films induced by D-limonene swelling. J Membr Sci 188: 39-48.

16. Tasselli F, Jansen JC, Sidari F, Drioli E (2005) Morphology and transport property control of modified poly(ether ether ketone) (PEEKWC) hollow fiber membranes prepared from PEEKWC/PVP blends: Influence of the relative humidity in the air gap. J Membr Sci 255: 13-22.

17. Feron PHM, Jansen AE (2002) CO2 separation with polyolefin membrane contactors and dedicated absorption liquids: Performances and prospects. Sep Purif Technol 27: 231-242.

18. Simon S, Espuche E (2014) Effect of different metal in situ growing routes on the morphology and gas separation properties of polyetherimide/palladium nanocomposite asymmetric membranes. Separation and Purification Technology 129: 41-49.
19. Chung TS, Jiang LY, Li Y, Kulprathipanja S (2005) Mixed matrix membranes (MMMs) comprising organic polymers with dispersed inorganic fillers for gas separation. Prog Polym Sci 32: 483-507.

20. Moore TT, Koros WJ (2005) Non-ideal effects in organic-inorganic materials for gas separation membranes. J Mol Struct 739: 87-98.

21. Merkel TC, Freeman BD, Spontak RJ, He Z, Pinnau I, et al., (2002) Ultrapermeable, reverse-selective nanocomposite membranes. Science 296: 519-522.

22. Slobodian P, Riha P, Lengalova A, Saha P (2011) Compressive stresselectrical conductivity characteristics of multiwall carbon nanotube networks. J Mater Sci 46: 3186-3190.

23. http://www.separationprocesses.com/Membrane/MT_Chp01c.htm.

24. Wang H, Dong X, Lin YS (2014) Highly stable bilayer MFI zeolite membranes for high temperature hydrogen separation. Journal of Membrane Science 450: 425-432. 